\title{
Hypocholesterolemic Effect of Tartary Buckwheat (F. tataricum Gaertn.) Extract from High Fat Diet Mice
}

\author{
Sung-Gyu Lee ${ }^{1}$, Dongsup Lee ${ }^{2}$ and Hyun Kang ${ }^{1, \dagger}$ \\ ${ }^{I}$ Department of Medical Laboratory Science, College of Health Science, Dankook University, \\ Cheonan-si, Chungnam 31116, Korea \\ ${ }^{2}$ Department of Clinical Laboratory Science, Hyejeon College, Choongchung, Hongseoung 32244, Korea
}

To investigate the efficacy of extract of Tartary buckwheat (F. tataricum Gaertn.) in high fat diet (HFD) in mice, the F. tataricum Gaertn. extract (FTE) was orally administered to mice with a HFD at $300 \mathrm{mg} / \mathrm{kg} / \mathrm{day}$ for 4 weeks. Our results show that FTE significantly inhibited fat accumulation. Moreover, FTE markedly reduced the final body weight with a decrease in epididymal adipose tissue mass and adipocyte size compared with the untreated HFD-induced group. Additionally, FTE ameliorated serum total cholesterol, triglyceride, and low-density lipoprotein cholesterol levels. The results show that Tartary buckwheat possesses hypocholesterolemic effect through downregulating lipid metabolism. Further studies are required in this area to strengthen the anti-obesity effects of FTE with active component, and it can be used a pro-drug instead of whole extract.

Key Words: Tartary buckwheat, Hypocholesterolemic, Lipid Metabolism, High fat diet Mice

There are 2 kinds of cultivated buckwheat, common buckwheat (Fagopyrum esculentum Moench) and tartary buckwheat (Fagopyrum tataricum Gaertn.) (Li et al., 2001). Buckwheat sprouts have presented that the sprouts contain several flavonoids, including orientin, isoorientin, vitexin, isovitexin, rutin, and quercetrin, whereas tartary buckwheat sprouts contain only rutin. Buckwheat is known for nutrient food due to supply of amino acids and protein (Zielinski et al., 2000). The nutritive values of the edible parts of sprouts of beans, crops, and vegetables have gained interest in recent years. Sprouts are recognized as outstanding dietary vegetables in Asia, Europe, and the United States. Sprouts are important source of protein, mineral, dietary fiber, and vitamins in human diets. Especially, the contents of polyphenols, which are secondary plant metabolites, have been increased or newly synthesized during sprouting. Polyphenols have attracted a great deal of attention due to their various health benefits for human (Quettier-Deleu et al., 2000). Tartary buckwheat seed is known to contain a large amount of rutin (Kitabayashi et al., 1995). Rutin and other flavonoids from buckwheat have beneficial effects for human health including hypolipidemic effect (Mukuda et al., 2001). The protein content of FTE was $46 \%$, and its amino acid composition of FTE was similar to that of common buckwheat protein product (Kitabayashi et al., 1995).

Twenty-four male C57BL/6J mice (age 5 6 weeks old) were obtained from DBL Inc. (Eumseong-gun, Republic of Korea). All the experimental animals were housed in a

* Received: November 29, 2016 / Revised: January 1, 2017 / Accepted: March 17, 2017

†Corresponding author: Hyun Kang. Department of Medical Laboratory Science, College of Health Science, Dankook University, Cheonan-si, Chungnam 31116, Korea.

Tel: +82-41-550-3015, Fax: +82-41-559-7934, e-mail: hkang@dankook.ac.kr

(C) The Korean Society for Biomedical Laboratory Sciences. All rights reserved.

(C) This is an Open Access article distributed under the terms of the Creative Commons Attribution Non-Commercial License (http://creativecommons.org/licenses/by-nc/3.0/) which permits unrestricted non-commercial use, distribution, and reproduction in any medium, provided the original work is properly cited. 
group $(n=6)$ and fed ad libitum water and food, under 12 $\mathrm{h}$ light and dark conditions. The Institutional Animal Ethics Committee guidelines were followed for handling animals in this study. All mice had free access to food and water. They were fed $10 \mathrm{kcal} \%$ low fat diet (Research diet, New Brunswick, NJ, USA) fat or $60 \mathrm{kcal} \%$ high fat diet (Research diet, New Brunswick, NJ, USA) throughout the 8-week treatment period. The animals were subdivided into four groups: $10 \%$ low fat diet (LF), $60 \%$ high fat diet (HF), HF + FTE $300 \mathrm{mg} / \mathrm{kg} /$ day (FTE300), and HF + green tea extract (GRE) $300 \mathrm{mg} / \mathrm{kg} /$ day (GRE300) and fed for 4 weeks. The body weight of rats was recorded once a week, whereas food intake was recorded daily. The food efficiency ratio (FER) (body weight gain per g consumed food) was calculated throughout the experiment. At the end of the 4-week treatment period after an overnight fast, rats were sacrificed under ether anesthesia, and blood was collected in a heparinized tube. The collected blood was centrifuged at 3,000 rpm for $15 \mathrm{~min}$, and the plasma was stored at $-70^{\circ} \mathrm{C}$ until processed. The liver, kidney, spleen, epididymis, epididymal fat, and abdominal fat were removed immediately, washed in ice-cold saline, and weighed. The concentration of plasma TC, HDL-cholesterol, and LDL-cholesterol were determined using a Konelab20XT automatic blood analyzer. The results were expressed as mean \pm standard deviation (S.D.) and statistically analyzed by analysis of variance (ANOVA). Duncan's multiple range test was performed to determine significant differences among the groups, and differences at $P<0.05$ were considered to be significant.

In order to study the hypocholesterolemic effect of FTE in vivo, we fed a high fat diet to mice for 4 weeks. According to the food efficiency ratio (FER) equation, a change in body weight is the most important factor affecting the FER, as there is no large change in the amount of ingested food. Thus, it is possible to apply the FER as an indicator; a small value for the FER can effectively predict the avoidance of obesity. Body weight gain in the HF group was higher than that of the LF, however, that of the FTE300 group was lower compared to the HF group $(P<0.05)$ (Fig. 1A). The food efficiency ratio (FER) of the HF group was significantly higher, however, that of the FTE300 group was lower compared to the HF group $(P<0.05)$ (Fig. 1B). Mice that were
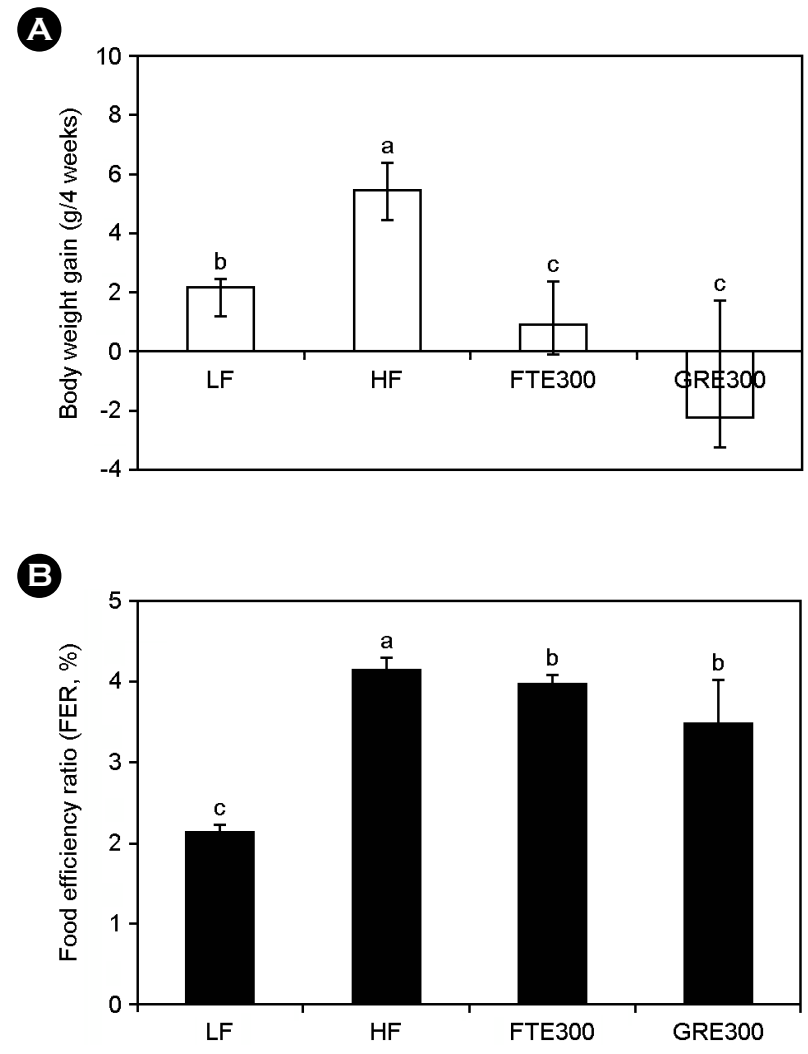

Fig. 1. Effect of FTE on body weight and food efficiency ratio (FER) in high fat diet (HF)-fed mice. (A) Effect of FTE on body weight gain in HF-fed mice. (B) Effect of FTE on FER in HF-fed mice. Food efficiency ratio (FER) = increased body weight $(\mathrm{g}) /$ food intake. The values are mean \pm S.D. $(n=6)$. Values with a common superscript letter within the same column are not significantly different $(P<0.05)$. LF: $10 \%$ low fat diet, HF: $60 \%$ high fat diet + vehicle, FTE300: $60 \%$ high fat diet + FTE, $300 \mathrm{mg} / \mathrm{kg}$, GRE300: $60 \%$ high fat diet + GRE, $300 \mathrm{mg} / \mathrm{kg}$.

orally administered FTE $(300 \mathrm{mg} / \mathrm{kg}$ ) with HF showed decreased body weight and food efficiency compared to HF-fed mice.

In our study, we speculated that the less body weight in FTE fed groups might be due to the satiety effect that results reduction in fat tissue weight which is related to body weight gain. However, we could not measure the fat and lean mass parameters in all treatment groups. Mice that were orally administered FTE with HF had a lower amount of both abdominal and epididymal fat compared to the HF group (Fig. 2). Abdominal fat weights of the HF group were significantly higher, compared to the LF group, and those of the FTE300 group were significantly lower compared to the 
A

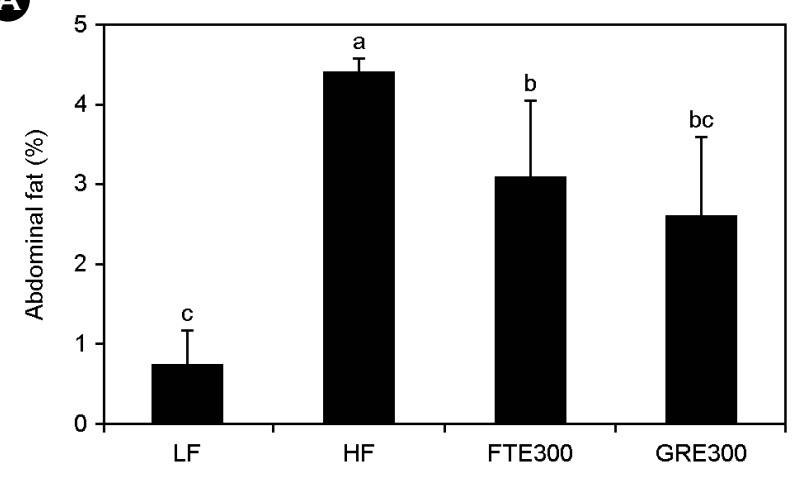

B

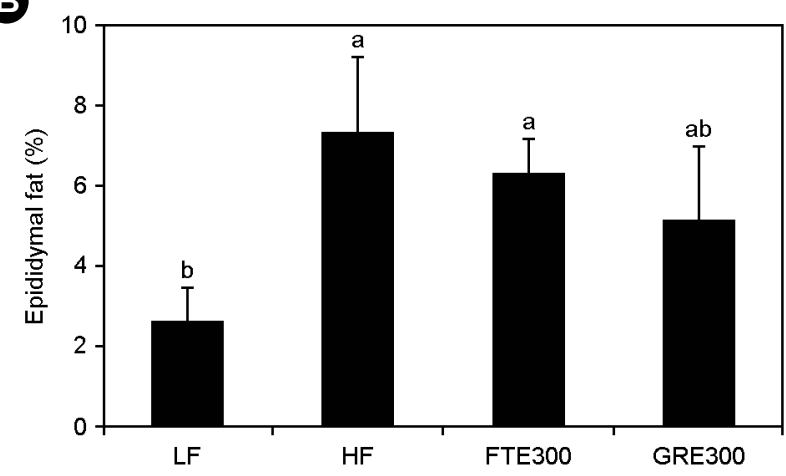

Fig. 2. Different adipose tissue weight in mice fed with experimental diet for 4 weeks. (A) Effect of FTE on abdominal fat weight in HF-fed mice. (B) Effect of FTE on epididymal fat weight in HF-fed mice. The values are mean \pm S.D. (n=6). Values with a common superscript letter within the same column are not significantly different $(P<0.05)$. LF: $10 \%$ low fat diet, HF: $60 \%$ high fat diet + vehicle, FTE300: 60\% high fat diet + FTE, $300 \mathrm{mg} / \mathrm{kg}$, GRE300: 60\% high fat diet + GRE, $300 \mathrm{mg} / \mathrm{kg}$.

Table 1. Effects of FTE on total cholesterol, HDL-cholesterol, and LDL-cholesterol mice fed a high fat diet for 4 weeks

\begin{tabular}{lccc}
\hline \hline & $\begin{array}{c}\text { Total cholesterol } \\
(\mathrm{mg} / \mathrm{dL})\end{array}$ & $\begin{array}{c}\text { HDL-cholesterol } \\
(\mathrm{mg} / \mathrm{dL})\end{array}$ & $\begin{array}{c}\text { LDL-cholesterol } \\
(\mathrm{mg} / \mathrm{dL})\end{array}$ \\
\hline LF & $124.00 \pm 2.65^{\mathrm{c}}$ & $15.12 \pm 7.01^{\mathrm{ab}}$ & $13.00 \pm 3.92^{\mathrm{c}}$ \\
HF & $169.67 \pm 5.51^{\mathrm{a}}$ & $16.45 \pm 0.13^{\mathrm{b}}$ & $32.67 \pm 4.04^{\mathrm{a}}$ \\
FTE300 & $150.00 \pm 5.45^{\mathrm{b}}$ & $20.55 \pm 3.27^{\mathrm{a}}$ & $21.00 \pm 2.21^{\mathrm{b}}$ \\
GRE300 & $152.67 \pm 27.43^{\mathrm{abc}}$ & $23.59 \pm 4.70^{\mathrm{a}}$ & $21.67 \pm 7.58^{\mathrm{abc}}$ \\
\hline
\end{tabular}

The values are mean \pm S.D. $(n=6)$. Values with a common superscript letter within the same column are not significantly different $(P<0.05)$. LF: 10\% low fat diet, HF: 60\% high fat diet + vehicle, FTE300: 60\% high fat diet + FTE, $300 \mathrm{mg} / \mathrm{kg}, \mathrm{GRE} 300: 60 \%$ high fat diet + GRE, $300 \mathrm{mg} / \mathrm{kg}$.

HF group $(P<0.05)$ (Fig. 2A). The effects of FTE on HFDinduced obesity were primarily responsible for inhibiting adipogenesis in adipose tissue and regulating lipid metabolism, such as through lipogenesis and fatty acid oxidation.

Tartary buckwheat is rich in flavonoids, including the rutin, quercetin 3-O-rutinoside-3'-O- $\beta$-glucopyranoside, kaempferol 3-O-rutinoside, and quercetin (Li et al., 2010). Using $\mathrm{C} 57 \mathrm{BL} / 6 \mathrm{~J}$ mice several authors have found reductions in body fat induced by Quercetin treatment. Liang et al. (2009) reported that mice fed on a HFD supplemented with Quercetin $(66 \mathrm{mg} / \mathrm{kg}$ body weight/d) were protected against weight gain induced by the diet. Moreover, Ohkoshi et al. (2007) showed a decrease in visceral and subcutaneous adipose tissue. Kobori et al. (2011) reported that chronic dietary intake of Quercetin reduced body weight gain, as well as visceral and liver fat accumulation, and improved systemic parameters related to metabolic syndrome (hyperglycemia, hyperinsulinemia and dyslipidemia), probably by decreasing oxidative stress and increasing PPAR $\alpha$ expression.

In the previous reports indicate that the active components of tartary buckwheat for reductions in body weight and fat can be considered as the quercetin.Results for serum lipid levels are shown in Table 1. The HF group showed a significant $(P<0.05)$ increase in serum total cholesterol and LDLcholesterol levels compared with the LF group. In contrast, FTE300 and GRE300 administration caused a significant $(P<0.05)$ decrease in serum total cholesterol and LDLcholesterol levels, compared to those in the HF group, while causing the reverse on serum HDL-cholesterol. Wang et al. (2009) reported the effects of tartary buckwheat bran extract on antioxidation status and on lipid profile in hyperlipidemic rats. In this study, 7-week-old male Wistar rats were fed a 
High fat diet to induce hyperlipemia. Tartary buckwheat bran extract was shown to significantly reduce triglycerides and cholesterol in the serum and liver of rats, raise serum antioxidant activity and inhibit serum lipid peroxide formation. Kuwabara et al. (2007) examined the effects of different types of buckwheat sprouts on the plasma cholesterol concentration, fecal steroid excretion and hepatic mRNA expression related to cholesterol metabolism in rats. The hypocholesterolemic effect of tartary buckwheat sprout powders was evident compared to the control diet in rats. It appears that the cholesterol-lowering effect of buckwheat sprout powder is dependent on fecal steroids, especially bile acid, excretion and cholesterol $7 \alpha$-hydroxylase mRNA.

Recent studies described tartary buckwheat protein, sprouts, leaves, flowers and bran have hypolipidemic activity. Additionally, tartary buckwheat is a good source of rutin, which has many potentially health-beneficial effects according to in vitro studies. In vitro tests have shown that rutin may inhibit LDL oxidation and HDL oxidation (Milde et al., 2004). Furthermore, FTE contains flavonoids, phenols, and phenolic might be responsible for lowering effect on lipids in HFD mice. Recently, much attention has been focused on the antioxidant function of these flavonoids and the mechanisms. Quercetin is also known to reduce high blood pressure and the risk of arteriosclerosis (Duarte et al., 2001). Rutin can suppress colon carcinogenesis (Volate et al., 2005). These physiological functions appear to be at least in part ascribed to their antioxidant functions.

In summary, this is first study to demonstrate that hypocholesterolemic effect of FTE by lipid metabolism from High fat diet mice. Our results show that FTE effects on reduction in the body weight gain and food intake, reducing lipid in adipose tissue, and increasing HDL with reducing TG and LDL in mice. This information presented in the current study suggests that FTE, including various flavonoids, might possibly have anti-obesity effects with hypocholesterolemic effect on mice.

\section{Acknowledgements}

This work (Grants No. C0267961) was supported by Business for Academic-industrial Cooperative establishments funded Korea Small and Medium Business Administration in 2015.

\section{Conflict of interest}

The authors declare that there is no conflict of interests regarding the publication this articles.

\section{REFERENCES}

Duarte J, Perez-Palencia R, Vargas F, Ocete MA, Perez-Vizcaino F, Zarzuelo A, Tamargo J. Antihypertensive effects of the flavonoid quercetin in spontaneously hypertensive rats. British Journal of Pharmacology. 2001. 133: 117-124.

Kitabayashi H, Ujihara A, Hirose T, Minami M. Variental differences and heritability for rutin content in common buckwheat, Fagopyrum esculentum Moench. Japanese Journal of Breeding. 1995. 45: 75-79.

Kobori M, Masumoto S, Akimoto Y, Oike H. Chronic dietary intake of quercetin alleviates hepatic fat accumulation associated with consumption of a Western-style diet in C57/BL6J mice. Molecular Nutrition \& Food Research. 2011. 55: 530 -540 .

Kuwabara T, Han KH, Hashimoto N, Yamauchi H, Shimada K, Sekikawa M. Tartary buckwheat sprout powder lowers plasma cholesterol level in rats. Journal of Nutritional Science and Vitaminology. 2007. 53: 501-507.

Liang $\mathrm{C}$, Oest ME, Prater ME. Intrauterine exposure to high saturated fat diet elevates risk of adult-onset chronic diseases in C57BL/6 Mice. Birth Defects Research (Part B). 2009. 86: 377-384.

Li D, Li X, Ding X. Composition and antioxidative properties of the flavonoid-rich fractions from tartary buckwheat grains. Food Science and Biotechnology. 2010. 19: 711-716.

Li SQ, Zhang HQ. Advances in the development of functional foods from buckwheat. Critical Reviews in Food Science and Nutrition. 2001. 41: 451-464.

Milde J, Elstner EF, Grassmann J. Synergistic inhibition of lowdensity lipoprotein oxidation by rutin, gamma-terpinene, and ascorbic acid. Phytomedicine. 2004. 11: 105-113.

Mukoda T, Sun B, Ishiguro A. Antioxidant activities of buckwheat hull extract toward various oxidative stress in vitro and in vivo. Biological and Pharmaceutical Bulletin. 2001. 24: 209 -213 .

Ohkoshi E, Miyazaki H, Shindo K. Constituents from the leaves of Nelumbo nucifera stimulate lipolysis in the white adipose 
tissue of mice. Planta Medica. 2007. 73: 1255-1259.

Quettier-Deleu C, Gressier B, Vasseur J, Dine T, Brunet C, Luyckx M. Phenolic compounds and antioxidant activities of buckwheat (Fagopyrum esculentum Moench) hulls and flour. Journal of Ethnopharmacology. 2000. 72: 35-42.

Tomotake H, Yamamoto N, Kitabayashi H, Kawakami A, Kayashita J, Ohinata H. Preparation of tartary buckwheat protein product and its improving effect on cholesterol metabolism in rats and mice fed cholesterol- enriched diet. Journal of Food Science. 2007. 72: 528-533.

Volate SR, Davenport DM, Muga SJ, Wargovich MJ. Modulation of aberrant crypt foci and apoptosis by dietary herbal supplements (quercetin, curcumin, silymarin, ginseng and rutin). Carcinogenesis. 2005. 26: 1450-1456.

Wang M, Liu JR, Gao JM, Parry JW, Wei YM. Antioxidant activity of Tartary buckwheat bran extract and its effect on the lipid profile of hyperlipidemic rats. Journal of Agricultural and Food Chemistry. 2009. 57: 5106-5112.

Zielinski H, Kozłowska H. Antioxidant activity and total phenolics in selected cereal grains and their different morphological fractions. Journal of Agricultural and Food Chemistry. 2000. 48: 2008-2016. 